\title{
VisuAlgo - Visualising Data Structures and Algorithms Through Animation
}

\author{
Steven HALIM \\ School of Computing, National University of Singapore \\ Computing 1, 13 Computing Drive, 117417, Singapore \\ e-mail:dcssh@nus.edu.sg
}

VisuAlgo (Fig. 1, http: / / visualgo.net) is the continuation of the work that was presented in IOI conference 3 years ago ${ }^{1}$ (Halim et al. 2012). VisuAlgo retains all the strong points of its predecessor:

- A web-based algorithm visualization tool without the need to install any additional software.

- It uses the latest web technology: HTML5, CSS3, JavaScript.

- It allows users to specify their own algorithm inputs and the visualization will work with that inputs.

- It is a collection of algorithm visualizations with unified interface.

VisuAlgo is a major improvement over its predecessor with 2000 sessions daily from worldwide visitors:

- It has significantly many more algorithm visualizations in the collection - all with the same unified look and feel. Almost all visualize-able data structures and algorithms covered in the author's Competitive Programming book $3^{\text {rd }}$ ed (Halim and Halim, 2013) have been included in VisuAlgo.

- It has an improved User Interface and more detailed algorithm animation steps.

- More importantly, we have added an important learning component: An Online Quiz tool (Fig. 2). It currently has hundreds of questions (and growing) with randomized inputs and/or question parameters that can be graded instantly. A Computer Science instructor can assess the basic data structure and algorithm knowledge of his/her students with much less effort. Computer Science students can also self-assess their proficiency of the basic material and they can always go back to corresponding visualization tool to restudy the concepts if they need to do so.

\footnotetext{
${ }^{1}$ The old URL: http://www. comp. nus.edu.sg/ stevenha/visualization/ is now no longer used.
} 


\section{References}

Halim, S., Koh, Z.C., Loh, B.H.V., Halim, F. (2012). Learning algorithms with unified and interactive webbased visualization. Olympiads in Informatics, 6, 53-68.

Halim, S., Halim, F. (2013). Competitive Programming 3: The New Lower Bound of Programming Contests. http: //cpbook. net

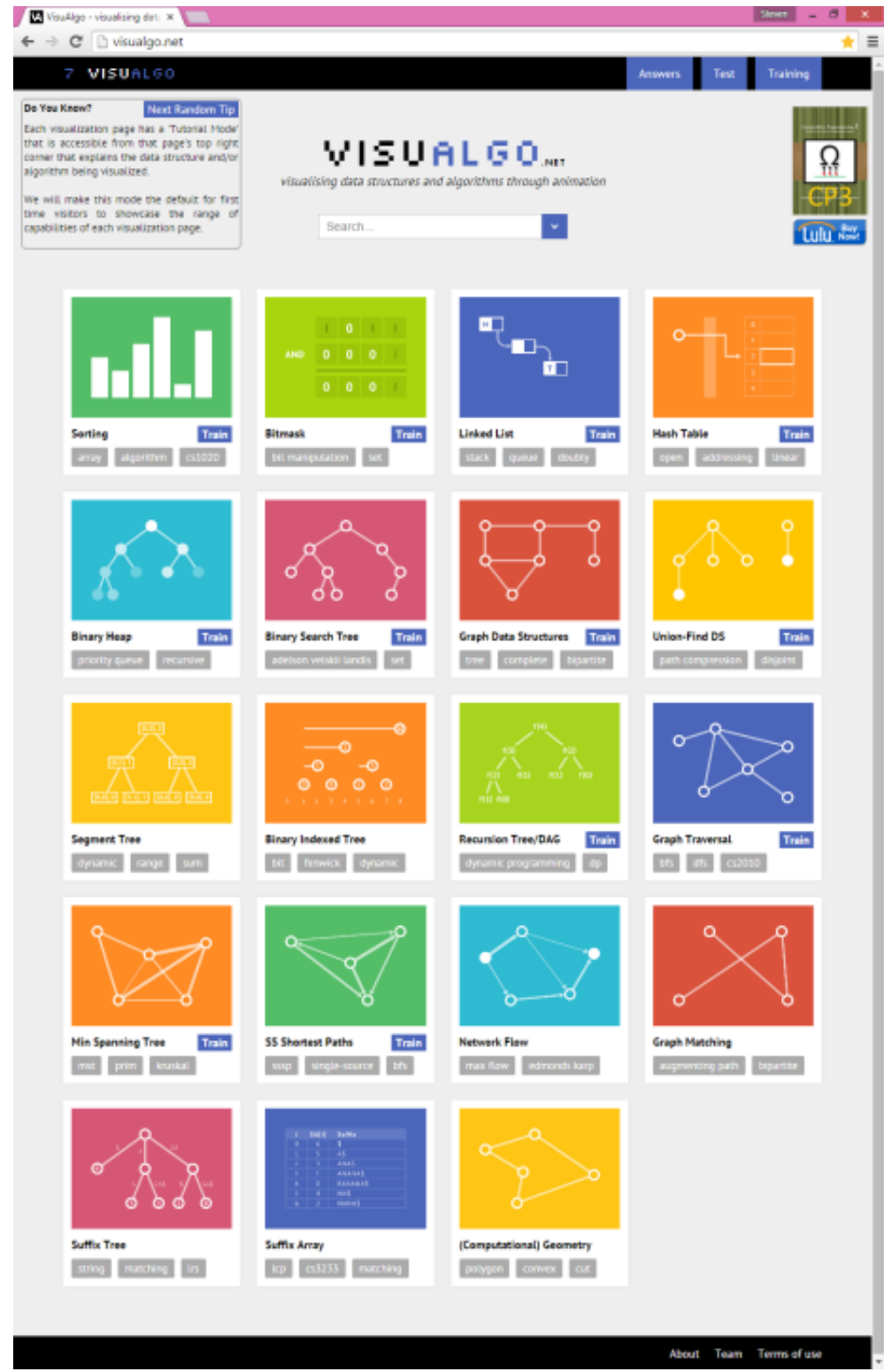

Fig. 1. VisuAlgo landing page. 


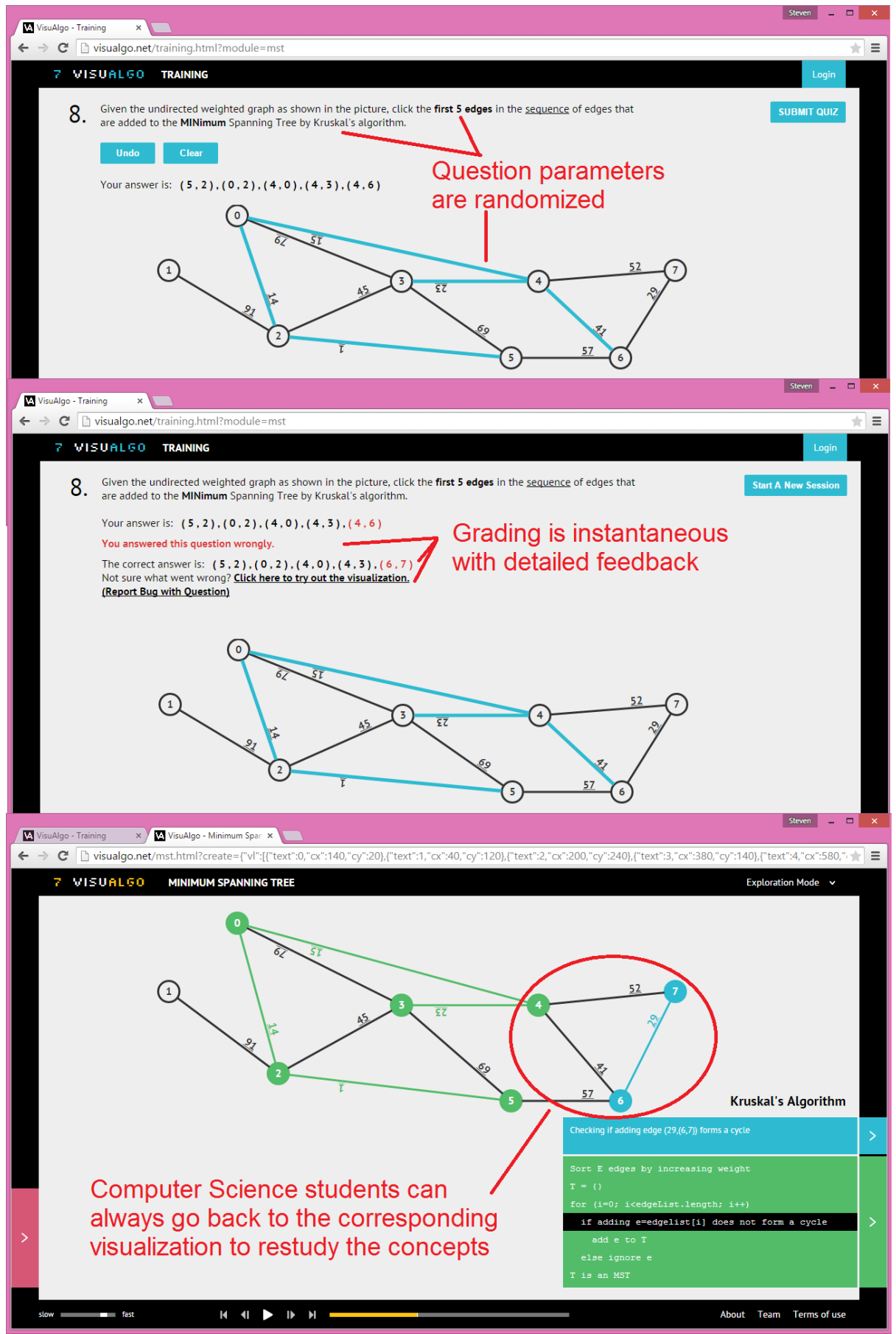

Fig. 2. The newest Online Quiz feature of VisuAlgo. 